\title{
RITA can induce cell death in p53-defective cells independently of p53 function via activation of JNK/ SAPK and $\mathrm{p} 38$
}

\author{
A Weilbacher ${ }^{1}$, M Gutekunst ${ }^{1}$, M Oren ${ }^{2}$, WE Aulitzky ${ }^{3}$ and $\mathrm{H}$ van der Kuip ${ }^{\star, 1}$
}

Significant advances have been made in the development of small molecules blocking the p53/MDM2 interaction. The Mdm2 inhibitor Nutlin-3 is restricted to tumors carrying wtp53. In contrast, RITA, a compound that binds p53, has recently been shown also to restore transcriptional functions of mtp53. As more than $50 \%$ of solid tumors carry p53 mutations, RITA promises to be a more effective therapeutic strategy than Nutlin-3. We investigated effects of RITA on apoptosis, cell cycle and induction of 45 p53 target genes in a panel of 14 cell lines from different tumor entities with different p53 status as well as primary lymphocytes and fibroblasts. Nine cell strains expressed wtp53, four harbored mtp53, and three were characterized by the loss of p53 protein. A significant induction of cell death upon RITA was observed in 7 of 16 cell lines. The nonmalignant cells in our panel were substantially less sensitive. We found that in contrast to Nultin-3, RITA is capable to induce cell death not only in tumor cells harboring wtp53 and mtp53 but also in p53-null cells. Importantly, whereas p53 has a central role for RITA-mediated effects in wtp53 cells, neither p53 nor p63 or p73 were essential for the RITA response in mtp53 or p53-null cells in our panel demonstrating that besides the known p53-dependent action of RITA in wtp53 cells, RITA can induce cell death also independently of p53 in cells harboring defective p53. We identified an important role of both p38 and JNK/SAPK for sensitivity to RITA in these cells leading to a typical caspase- and BAX/BAK-dependent mitochondrial apoptosis. In conclusion, our data demonstrate that RITA can induce apoptosis through p38 and JNK/SAPK not only in tumor cells harboring wtp53 and mtp53 but also in p53-null cells, making RITA an interesting tumor-selective drug.

Cell Death and Disease (2014) 5, e1318; doi:10.1038/cddis.2014.284; published online 10 July 2014

The p53 protein is activated by a variety of cellular stresses such as genotoxic damages, oncogenic activation and hypoxia leading to transcriptional activation of pro-apoptotic and cell cycle arrest genes, ${ }^{1,2}$ to transcriptional repression of anti-apoptotic and growth-promoting genes, ${ }^{3}$ and to direct binding of $\mathrm{p} 53$ to $\mathrm{BCL}-2$ family proteins triggering apoptosis in a transcription-independent manner. ${ }^{4-6}$ These activities are central for maintaining genetic stability and make p53 a classical key tumor suppressor. ${ }^{7,8}$ In fact, approximately half of all human cancers harbor mutations in the TP53 gene leading to loss of tumor suppressor function and/or gain of new oncogenic activities. ${ }^{9-12}$ In tumors without TP53 mutations, the p53 signaling pathway is frequently attenuated, for example, through amplifications of MDM2. ${ }^{13}$ Inactivation of normal p53 functions either by mutations or pathway alterations not only promotes tumorigenesis but also enables cancer cells to evade genotoxic stress-induced death.

Consequently, strong efforts have been made toward identification of small molecules capable of stabilizing wildtype p53 (wtp53) or reactivating normal p53 functions in cells harboring mutant p53 (mtp53). Solution approaches include inhibitors that interrupt the p53/MDM2 interaction or small molecules that bind mtp53 and restore wtp53 activity. ${ }^{14,15}$ Activation of wtp53 can be achieved by MDM2 inhibitors which interact with the p53-binding pocket subsequently inhibiting p53 degradation, such as Nutlins ${ }^{16,17}$ and MI- $43,{ }^{18}$ or by small molecules directly binding p53 and thereby impeding p53/ MDM2 interaction such as the thiophene derivate RITA (reactivation of p53 and induction of tumor cell apoptosis). ${ }^{19}$ RITA was identified as a p53-interacting molecule in a screen for compounds with growth-suppressive effects in cancer cells expressing wtp53 but not in their p53-null counterparts. ${ }^{19}$ RITA binds to the $N$-terminal p53 domain, probably leading to conformational changes thus impeding p53/MDM2 interaction. ${ }^{19}$ Interestingly, besides the fact that Nutlins and RITA target the same interaction (p53/MDM2), the effects on p53 activity are different. RITA-activated p53 predominantly affects apoptosis-related genes, whereas Nutlins mainly induce cell cycle regulators. ${ }^{20}$ These differential responses can be partially explained by RITA-mediated conformational changes of p53 and/or

\footnotetext{
${ }^{1}$ Dr Margarete Fischer-Bosch Institute of Clinical Pharmacology and University of Tuebingen, Auerbachstrasse 112, Stuttgart, Germany; ${ }^{2}$ Department of Molecular Cell Biology, Weizmann Institute of Science, Rehovot, Israel and ${ }^{3}$ Second Department of Internal Medicine, Robert-Bosch-Hospital, Auerbachstrasse 110, Stuttgart, Germany

${ }^{*}$ Corresponding author: $\mathrm{H}$ van der Kuip, Dr Margarete Fischer-Bosch Institute of Clinical Pharmacology, Auerbachstrasse 112, 70376 Stuttgart, Germany. Tel: +49 7118101 3730; Fax: +49 711 859295; E-mail: heiko.van-der-kuip@ikp-stuttgart.de

Abbreviations: BH3, Bcl-2 homology domain 3; BrdU, 2-Bromo-5-desoxyuridin; MOMP, mitochondrial outer membrane permeabilization; mt, mutant; PBMNC, peripheral blood mononuclear cell; RITA, reactivation of p53 and induction of tumor cell apoptosis, NSC652287; ROS, reactive oxygen species; siRNA, small interfering RNA; TMRM, tetramethylrhodamine methyl ester; wt, wild type

Received 19.2.14; revised 30.5.14; accepted 05.6.14; Edited by U Moll
} 
altered E3 ligase activities of Nutlin-bound MDM2. Recently, RITA was shown to reactivate mtp53 in human tumor cells. ${ }^{21}$

In the present study, we used a panel of cancer cell lines and nonmalignant cells with different p53 status. We found that in RITA-sensitive cells, RITA induces a p53-dependent cell death in wtp53 cells, whereas in cells carrying mtp53, RITA can also induce apoptosis independently of p53. This was confirmed by the finding that even in a p53-null cell line, RITA effectively induced apoptosis. RITA-induced apoptosis was partially mediated by the p38 and JNK/SAPK pathway.

\section{Results}

In contrast to Nutlin-3, effects of RITA on cell cycle and apoptosis are not strictly linked to p53 status. To evaluate effects of RITA in comparison with Nutlin-3 on induction of cell death and cell cycle in dependency of the p53 status, we selected a panel of cell lines from different tumor entities with wtp53, mtp53 or p53-null (Supplementary Table 1) as well as fibroblasts and PBMNCs from healthy donors. As expected and consistent with previous reports, ${ }^{17}$ response to Nutlin-3 was exclusively observed in wtp53 cells (Figure 1, left panel). Induction of cell death was seen in the embryonal carcinoma cell line NTERA-2D1, which has been demonstrated to exhibit a unique sensitivity toward p53 activation $^{22}$ and in the NSCLC cell line H460 (Figure 1a, left panel). Most cells including fibroblast and lymphocytes responded by $\mathrm{G} 1$ arrest (Figure $1 \mathrm{~b}$, left panel).

In contrast to Nutlin-3, RITA did not induce cell cycle arrest in the cell line panel used in this study (Figure 1b, right panel). However, significant induction of cell death was observed in 7 of 11 tumor cell lines (Figure 1a, right panel). Consistent with recently published studies, ${ }^{23}$ there was only marginal induction of cell death in wtp53-expressing nonmalignant cells $(<15 \%)$. Unexpectedly, in tumor cell lines, induction of cell death was not restricted to cells harboring wtp53 or mtp53 but could also be detected in the p53-null cell line OVCAR5 (Figure 1a, right panel), indicating that RITA can also be active in the absence of p53. Remarkably, sensitivity to RITAinduced cell death was divided into two distinct groups: highly sensitive cells with an induction of more than $60 \%$ cell death and cells with low sensitivity ( $<15 \%$ cell death). In contrast, the degree of sensitivity to Cisplatin was distributed equally in the same cell line panel (Supplementary Figure 1).

These data demonstrate that Nutlin-3 selectively affects wtp53 cells, whereas RITA can induce cell death also in cells harboring mtp53 or no p53.

Regulation of p53 targets by RITA is not correlated to the p53 status in the cell line panel investigated. To investigate activation of p53 as transcription factor in cells treated with RITA or Nutlin-3, we quantified expression of 45 bona fide p53 transcriptional targets (Supplementary Table 2$)^{2,24}$ by TaqMan-based real-time PCR. As expected, differential expression of p53 targets upon Nutlin-3 was predominantly observed in wtp53 cells, whereas mtp53 or p53-null cells showed only minor alterations. Consequently, cells clustered according to their p53 status (Figure 2a). Nineteen genes were significantly regulated by Nutlin-3 in cell strains harboring wtp53 (Benjamini-Hochberg-adjusted a
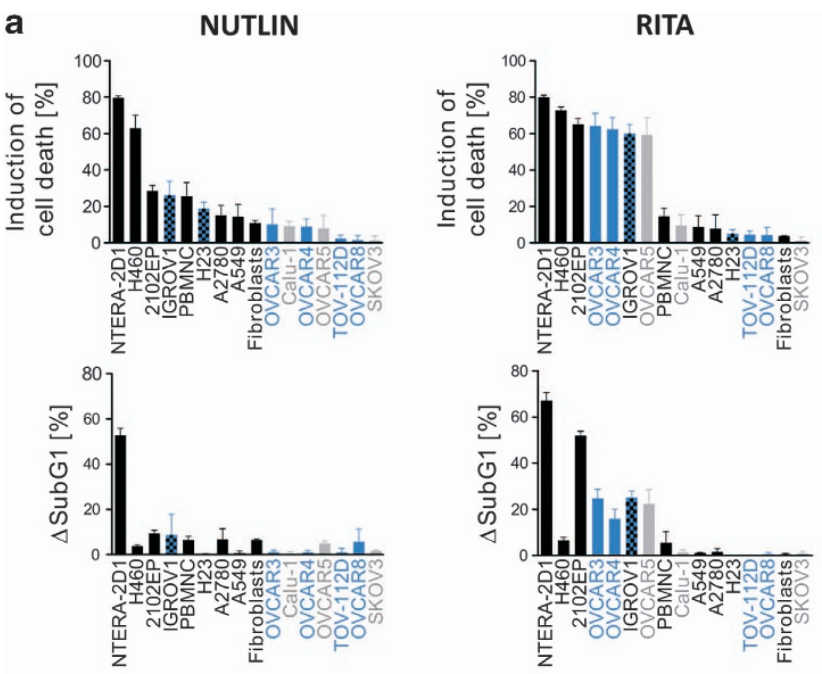

b
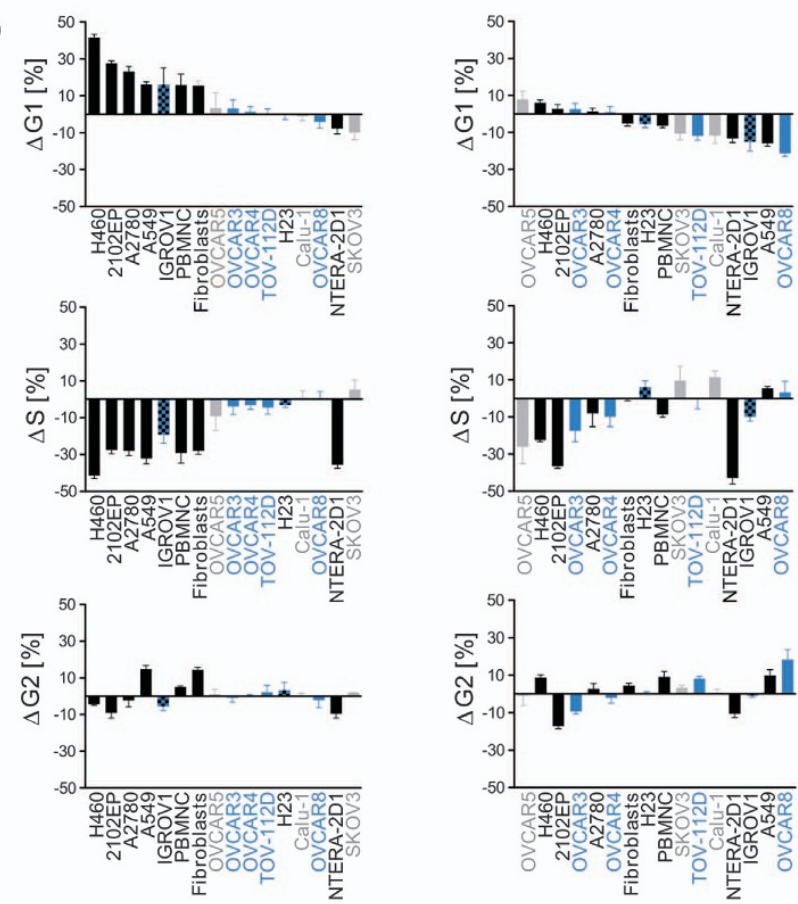

Figure 1 In contrast to Nultin-3, effects of RITA on cell cycle distribution and cell death is not strictly dependent on p53 status. A panel of cell lines from different tumor entities with different $p 53$ status and nonmalignant controls were incubated with/without Nutlin-3 (10 $\mu \mathrm{M}$; left) or RITA ( $1 \mu \mathrm{M}$; right) for $48 \mathrm{~h}$. Bars are colored according to the p53 status of the cells (wtp53: black bars, mtp53: blue bars, heterozygous cells: blue- and black-patterned bars). (a) In contrast to Nultin-3, induction of cell death by RITA is not restricted to wtp53 harboring cells. Cells were stained with AnnexinV-FITC/PI (upper panel) or PI and analyzed by flow cytometry. Graphs reflect percentages of induction of Annexin-positive cells (upper panel) and sub-G1 phase cells (lower panel) in RITA-treated samples. Values reflect means \pm S.D. from three independent experiments. (b) In contrast to Nutlin-3, RITA does not induce cell cycle arrest in the cell lines investigated. Cells were pulsetreated with BrdU, stained with an anti-BrdU-FITC antibody and PI, and analyzed by flow cytometry. Bars reflect the differences between the percentages of cells in G1, $S$ and G2/M phase after RITA treatment and untreated controls. Values reflect means \pm S.D. from three independent experiments

paired $t$-test $P<0.05, F C>2$; Supplementary Table 3). All 19 genes were found to be upregulated. We found no significantly regulated genes in p53-defective cells. 


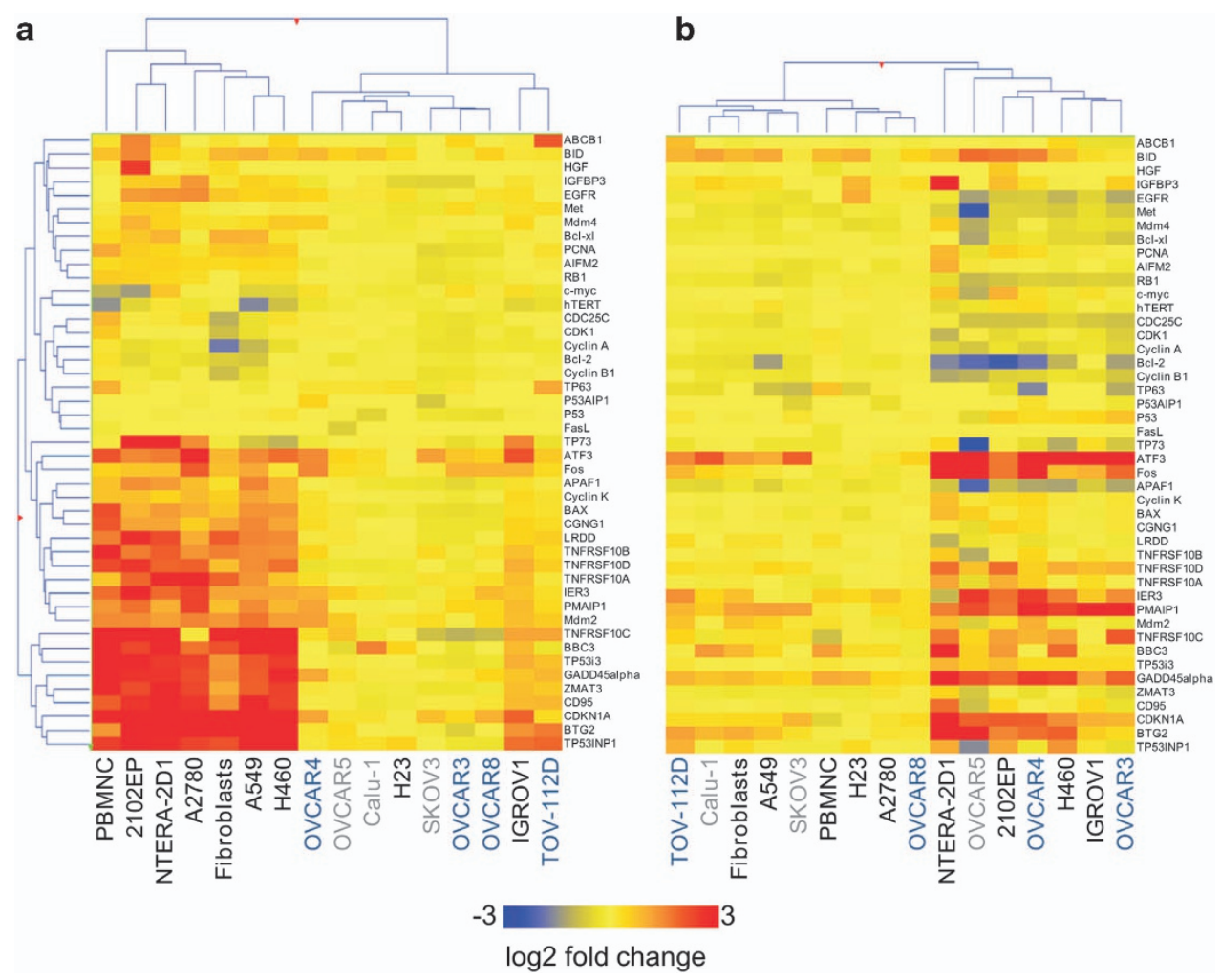

Figure 2 RITA-mediated p53 target gene induction is not correlated to p53 status in the cell line panel investigated. For gene expression analysis, cells were either treated with Nutlin-3 (a) or RITA (b) for $8 \mathrm{~h}$. Cells were then harvested and RNA was isolated. After cDNA synthesis and specific target gene amplification, gene expression of 45 bona fide p53 target genes was analyzed on the BioMark HD System. $\Delta \Delta$ Ct values were calculated and cluster analysis was accomplished by Euclidean similarity measurements combined with a complete linkage rule. Clustering was performed both on entities and conditions in the case of Nutlin-3. Order of genes after clustering was extracted and used for the clustering of RITA-treated samples. For RITA, clustering was performed for conditions. Indicated colors reflect means of log2 $\Delta \Delta \mathrm{Ct}$-values from three independent experiments

In contrast, RITA led to a differential regulation of p53 target genes predominantly in RITA-sensitive cells independent of their p53 status resulting in a clustering according to the RITA-sensitivity phenotype (Figure $2 b$ ). We found 10 genes upregulated and 3 genes downregulated in cells responding to RITA (Benjamini-Hochberg-adjusted paired $t$-test $P<0.05, F C>2$; Supplementary Table 3), whereas in nonresponding cells, there was only one gene significantly upregulated upon RITA (ATF3). It is noteworthy that PBMNCs and fibroblasts belonged to the second group showing only minor regulations of p53 target genes.

These data demonstrate that RITA can alter expression of p53 target genes independently of the p53 status.

RITA can induce cell death independently of p53 in mtp53 cells. Recently published data indicate that induction of cell death by RITA in mtp53 cells is due to reactivation of p53. ${ }^{21}$ We therefore next investigated whether mtp53 is necessary for RITA-dependent cell death in two RITAsensitive ovarian cancer cell lines of our panel harboring mtp53 (OVCAR3 and OVCAR4) by performing TP53 siRNA experiments. Knockdown of mtp53 was efficient in both cell lines (Figure 3, upper panels). We first evaluated possible effects of p53 silencing on RITA-mediated regulation of 45 typical p53 targets in these cells. We searched for those transcripts that were at least twofold differentially regulated upon RITA in cells pretreated with control siRNA or TP53
siRNA. Importantly, silencing of TP53 had no obvious effect on expression of p53 targets, including p21 (CDKN1A), NOXA (PMAIP1), GADD45 $\alpha, C-M Y C$ and BCL-2, which are known to be regulated upon RITA treatment ${ }^{20,21,25-27}$ (Figure 3, middle panels). There were only 2 out of 45 genes with at least twofold difference in expression in p53-silenced cells, namely TP63 in OVCAR3 (Figure 3, left middle panel) and $B B C 3$ in OVCAR4 (Figure 3 , right middle panel). Furthermore, silencing of TP53 had no effect on induction of RITA-induced cell death (Figure 3, lower panels). In contrast and in agreement with previously published data, ${ }^{19}$ RITA-induced cell death in wtp53-expressing cells was efficiently rescued by TP53 silencing (Supplementary Figure 2A). Of note, Pifithrin-alpha, a compound supposed to specifically block transcriptional p53 activity, almost completely rescued RITA-induced cell death not only in cells harboring mtp53 but also in the p53-null cell line OVCAR5 demonstrating that this effect is independent of p53 inhibition (Supplementary Figure 2B).

We therefore conclude that besides the known p53-dependent action of RITA in wtp53 cells, RITA can induce cell death also independently of p53 in cells harboring mtp53.

RITA-induced cell death in the p53-null cell line OVCAR5 is independent of $p 63$ and p73. Based on the fact that the transactivation domain, the DNA-binding domain and the 

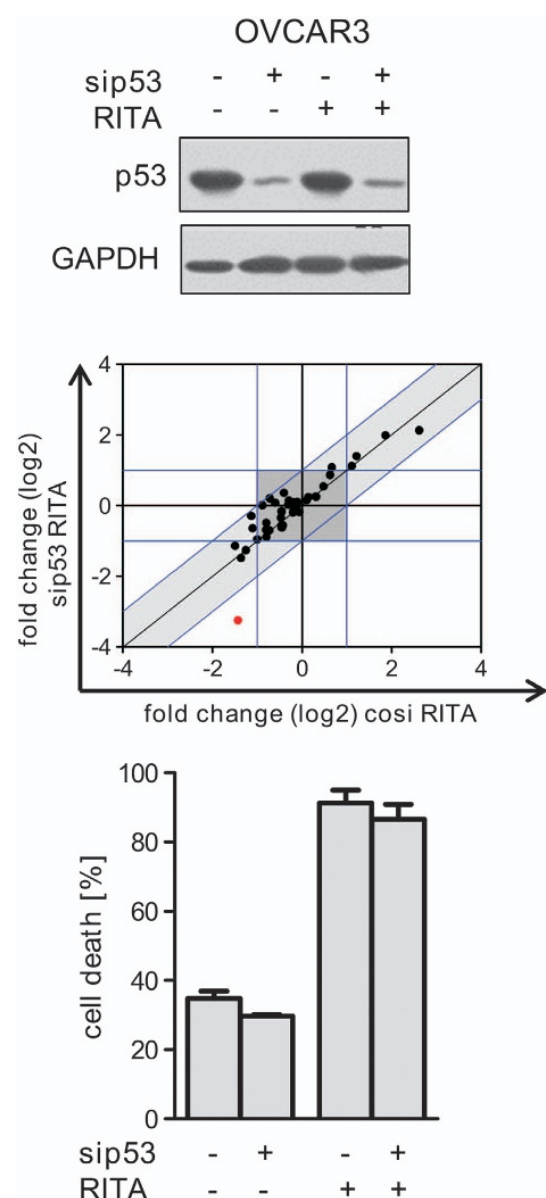
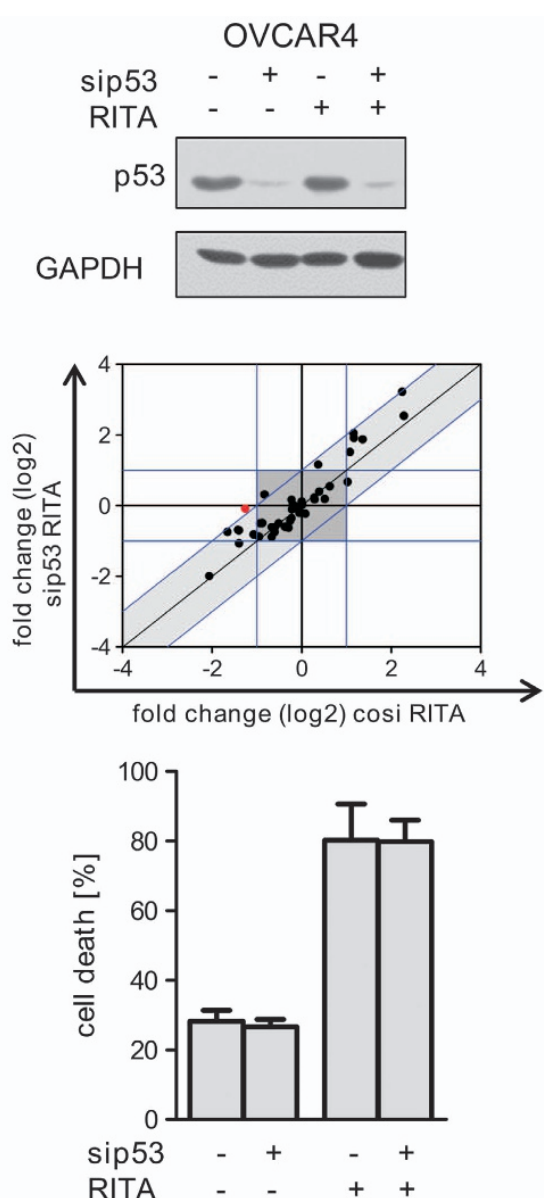

Figure 3 RITA can induce cell death independent of p53 in ovarian cancer cell lines harboring mtp53. OVCAR3 and OVCAR4 cells were used for TP53 knockdown analysis. Upper panel: representative p53 western blot analysis demonstrating knockdown efficacy upon siRNA treatment. Middle panel: RITA-mediated induction of 45 p53 target genes. Cells were incubated with/without RITA for $8 \mathrm{~h}$, total RNA was isolated and gene expression analysis was performed using specific assays for 45 bona fide p53 targets (Supplementary Table 2). Graph shows log2 $\Delta \Delta$ Ct-values of control siRNA ( $X$ axis) against the log2 $\Delta \Delta$ Ct-values from sip53-transfected cells ( $Y$ axis). Each dot represents mean values of one p53 target gene from three independent experiments. Genes with less than twofold differential expression upon RITA treatment are located within the dark gray rectangle. Genes with more than twofold differential regulation upon RITA but less than twofold difference in cells pretreated with control or TP53 siRNA are located in the light gray area. Genes with a more than twofold differential regulation and more than twofold difference are marked with red dots. Lower panel: cells were incubated with/without RITA for $48 \mathrm{~h}$, stained with AnnexinV-FITC/PI or TMRM and analyzed by flow cytometry. Graphs reflect means \pm S.D. from three experiments

oligomerization domain are highly conserved between p53, p63 and $p 73^{28}$ and all three share a number of target genes, ${ }^{29,30}$ we examined if $p 63$ or p73 may be involved in RITA-mediated regulation of p53 targets and cell death. We therefore silenced TP63 and TP73 in the p53-null cell line OVCAR5. As p63 protein was undetectable in OVCAR5 cells (not shown), we checked knockdown efficacy on mRNA level for both genes as well as on protein level for p73. Knockdown of both TP63 and TP73 as well as double knockdown was efficient (Figure 4, upper panel). Interestingly, neither silencing of TP63 or TP73 alone nor TP63/TP73 double knockdown showed significant effects on regulation of the p53 targets (Figure 4, middle panel). Furthermore, no changes in RITA-mediated cell death were observed upon single or double knockdowns of TP63 and TP73 (Figure 4, lower panel). A similar result was observed in the mtp53 cell line OVCAR4 (Supplementary Figure 3).

Therefore, RITA can induce cell death independently of p53, p63 and p73.
RITA induces a caspase-dependent mitochondrial cell death in mutant and p53-null cells. It has been demonstrated recently that RITA induces a caspase-dependent apoptosis in wtp53-expressing multiple myeloma cells. ${ }^{23}$ We therefore asked if the same is true for RITA-sensitive cells harboring mtp53 or p53-null from our panel. Indeed, pre-treatment with the pan-caspase inhibitor zVAD-FMK completely abrogated RITA-induced cell death in OVCAR3 and OVCAR4 (mtp53) as well as in the p53-null cell line OVCAR5 (Figure 5a).

To further analyze the role of the two pore-forming proteins $B A X$ and BAK for RITA-induced cell death, we performed siRNA-mediated $B A X$ and $B A K$ knockdowns in the mtp53 cell line OVCAR4 (Figure 5b, upper panel) and the p53-null cell line OVCAR5 (Figure 5c, upper panel). Simultaneous silencing of BAX and BAK led to an almost complete reduction of RITA-mediated cell death in the two cell lines (Figures $5 b$ and $c$, right panel). Of note, knockdown of $B A K$ alone was sufficient to partially rescue p53-null cells from RITA-induced apoptosis (Figure 5c, lower panel). 
p63
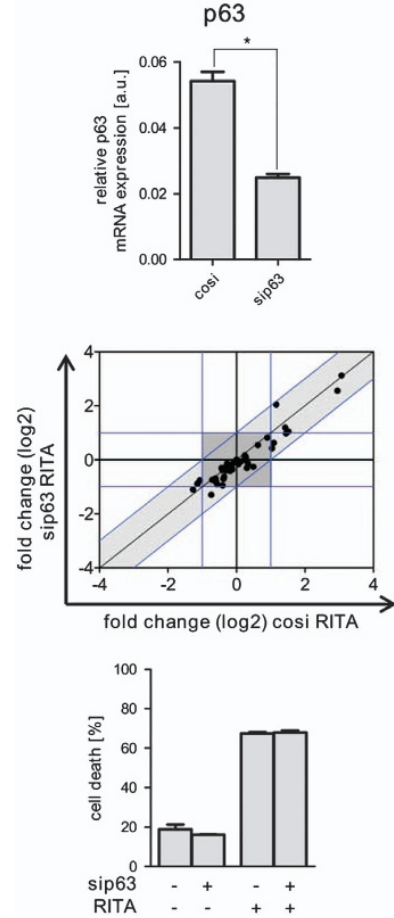

p73
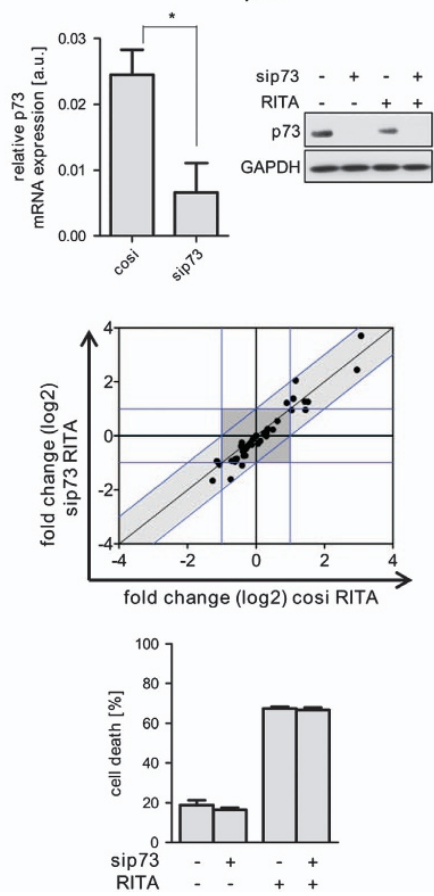
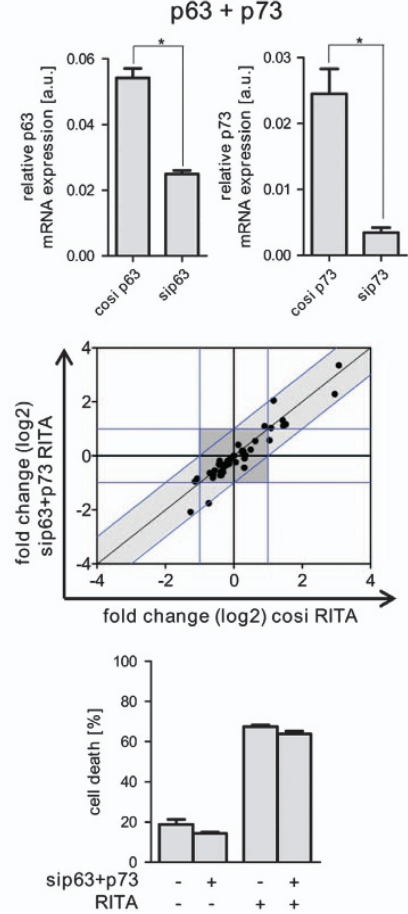

Figure 4 RITA can induce cell death independent of $p 63$ and p73 in the p53-null cell line OVACR5. Upper panel: knockdown efficacy was evaluated by real-time PCR using assays for TP63 and TP73 (Supplementary Table 2) and by western blot for p73. Graphs reflect means of three independent experiments \pm S.D. Middle panel: RITAmediated induction of $45 \mathrm{p} 53$ target genes. Cells were incubated with/without RITA for $8 \mathrm{~h}$, total RNA was isolated and gene expression analysis was performed using specific assays for 45 bona fide p53 targets (Supplementary Table 2). Graph shows log2 $\Delta \Delta \mathrm{Ct}$-values of control siRNA (X axis) against the log2 $\Delta \Delta \mathrm{Ct}$-values from sip53-transfected cells $(Y$ axis). Each dot represents mean values of one p53 target gene from three independent experiments. Genes with less than twofold differential expression upon RITA treatment are located within the dark gray rectangle. Genes with more than twofold differential regulation upon RITA but less than twofold difference in cells pretreated with control or TP53 siRNA are located in the light gray area. Lower panel: cells were incubated with/without RITA for $48 \mathrm{~h}$, stained with AnnexinV-FITC/PI and analyzed by flow cytometry. Graphs reflect means \pm S.D. from three experiments

Together, these data demonstrate that RITA can induce a caspase- and BAX/BAK-dependent mitochondrial apoptosis in mtp53 and p53-null cells.

RITA leads to reduced expression of anti-apoptotic Bcl-2 proteins and mitochondrial membrane depolarization. In RITA-sensitive cells, NOXA (PMAIP1) and PUMA (BBC3) were upon the most significantly regulated genes after RITA treatment (Supplementary Table 3). We therefore investigated the role of these pro-apoptotic proteins for RITA-induced apoptosis and performed silencing experiments using siRNAs against NOXA and PUMA. Despite effective downregulation of both pro-apoptotic proteins, neither NOXA nor PUMA had a significant impact on RITA-induced apoptosis (Figure 6a).

Of note, the gene coding for the anti-apoptotic BCL-2 protein was significantly downregulated upon RITA in RITA-sensitive cell lines (Supplementary Table 3). To deeply investigate the role of BCL-2, we analyzed BCL-2 protein levels in the mtp53 cell line OVCAR4 and in the p53-null cell line OVCAR5. As shown in Figure 6b, BCL-2 was reduced upon RITA in OVCAR4. Due to the fact that BCL-2 protein was undetectable in OVCAR5, we also investigated the levels of MCL-1, BCL-XL and BCL-W. Treatment with RITA reduced $M C L-1$ in both cell lines and BCL-xL in OVCAR4 indicating that at least one anti-apoptotic BCL-2 family member was reduced in response to treatment with RITA. The potential role of $\mathrm{BCL}-2$ and $\mathrm{MCL}-1$ was confirmed by the finding that the $\mathrm{BH} 3$ mimetic $\mathrm{ABT}-737$ had synergistic effects with RITA in RITA-sensitive cell lines (Figure 6c).

RITA mediates apoptosis via activation of p38 and JNK/ SAPK in p53-defective cells. It has been demonstrated that RITA induces JNK in multiple myeloma cells. ${ }^{31}$ We therefore next investigated whether JNK/SAPK is responsible for RITA-induced apoptosis in the ovarian carcinoma cell lines OVCAR3 (mtp53) and OVCAR5 (p53-null). We found that RITA indeed induced C-JUN phosphorylation, which was completely abrogated by SP600125 (Figure 7a, left). Concurrently, inhibition of RITA-mediated JNK/SAPK activation by SP600125 significantly blocked induction of apoptosis (Figure $7 \mathrm{a}$, middle and right). The role of JNK/SAPK for RITA-induced apoptosis was further confirmed by RNAi experiments. Interestingly, silencing of JNK1 was sufficient to reduce both c-JUN phosphorylation and RITA-mediated apoptosis by a degree comparable with that observed after pharmacological inhibition (Figure 7b), indicating a predominant role of this particular JNK protein. We also investigated the role of p38 for RITA-induced apoptosis in mtp53harboring cells. As shown in Figure 7c, pharmacological inhibition of p38 also reduced RITA-mediated c-JUN phosphorylation and RITA-induced apoptosis, although not that pronounced as observed upon inhibition of the JNK/SAPK pathway. 

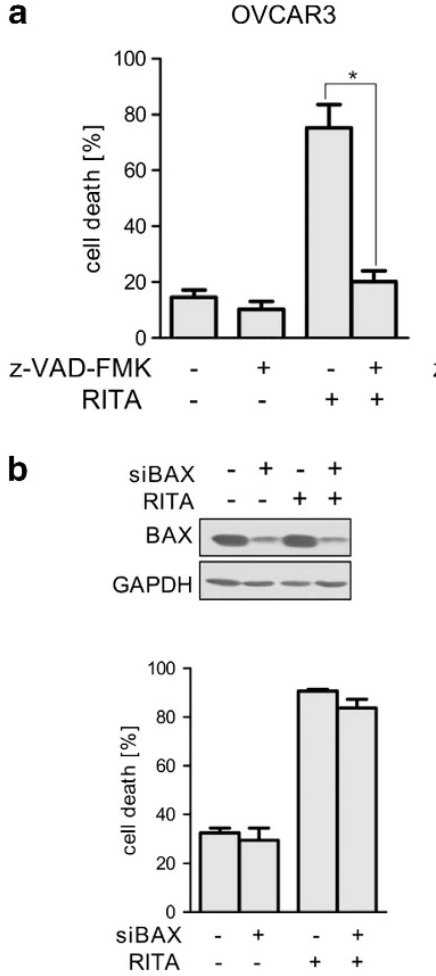

c
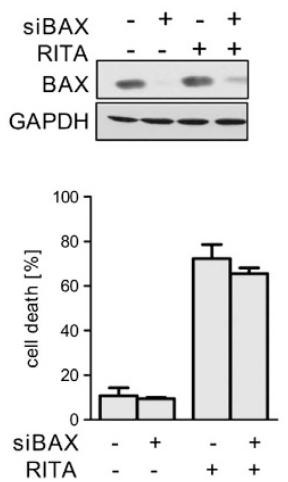

OVCAR4
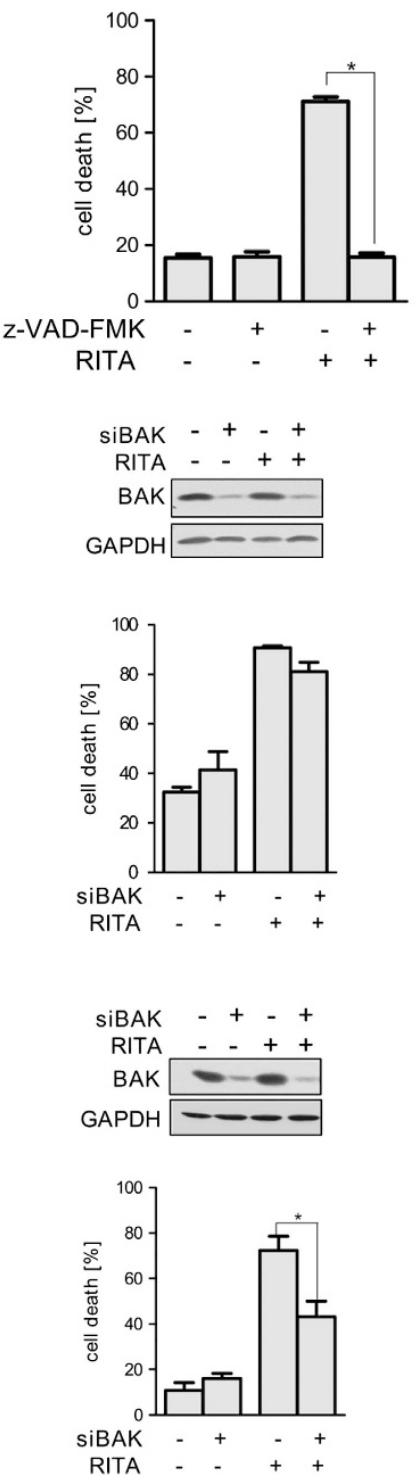

OVCAR5

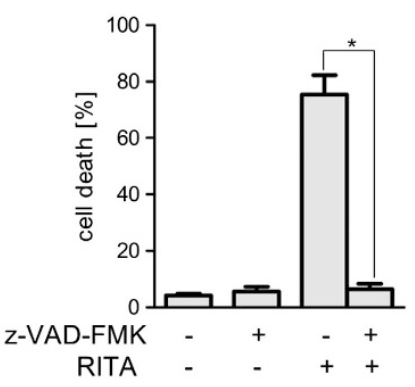

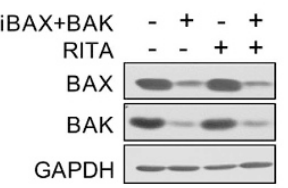
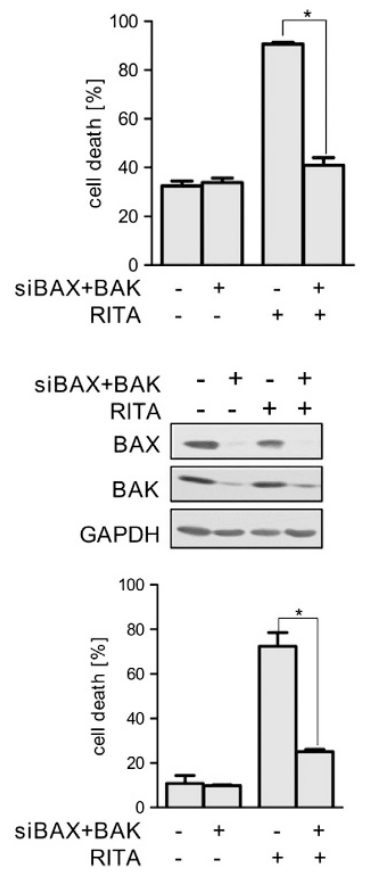

Figure 5 RITA induces caspase-dependent mitochondrial apoptosis in p53-deficient ovarian cancer cells. (a) Dependency of RITA-induced cell death on caspase activation. OVCAR3 (left), OVCAR4 (middle) and OVCAR5 (right) cells were pre-incubated with/without zVAD-FMK $2 \mathrm{~h}$ before RITA treatment. After $48 \mathrm{~h}$, cells were stained with AnnexinV-FITC/PI and analyzed by flow cytometry. Graphs reflect means \pm S.D. from three experiments. (b) Dependency of RITA-induced MOMP on the pore-forming complex proteins BAX and BAK in the mtp53 ovarian cancer cell line OVCAR4. Upper panel: representative BAX and BAK western blot analyses demonstrating knockdown efficacy upon siRNA treatment. Lower panel: cells were pre-incubated with/without indicated siRNAs $48 \mathrm{~h}$ before starting with RITA treatment for another $48 \mathrm{~h}$. Cells were stained for TMRM and analyzed by flow cytometry. Graphs reflect means \pm S.D. from three experiments. (c) Dependency of RITA-induced apoptosis on the pore-forming complex proteins BAX and BAK in the p53-null ovarian cancer cell line OVCAR5. Upper panel: representative BAX and BAK western blot analyses demonstrating knockdown efficacy upon siRNA treatment. Lower panel: cells were pre-incubated with/without indicated siRNAs $48 \mathrm{~h}$ before starting with RITA for another $48 \mathrm{~h}$, stained with AnnexinVFITC/PI and analyzed by flow cytometry. Graphs reflect means \pm S.D. from three experiments

Together, these data indicate that JNK/SAPK and p38 have an important role for RITA-induced apoptosis in p53-deficient cells.

\section{Discussion}

We demonstrate that sensitivity of cancer cells to RITA is not strictly linked to their p53 status. RITA is capable to induce apoptosis not only in cells harboring wtp53 or mtp53 but also in p53-null cells. In these cells, RITA induced a p53-independent mitochondrial cell death predominantly mediated by the JNK/SAPK and p38 MAPK pathways.

We analyzed a panel of cell lines harboring different functional states of p53. In these cell lines, we observed a remarkably diverse distribution of RITA-mediated functional and molecular effects without an obvious dependency on the p53 status. We observed two distinctive phenotypes regarding RITA sensitivity: cells with pronounced induction of apoptosis (more than 60\%) and cells showing no obvious response (less than 15\%). Within the group of responding cell 

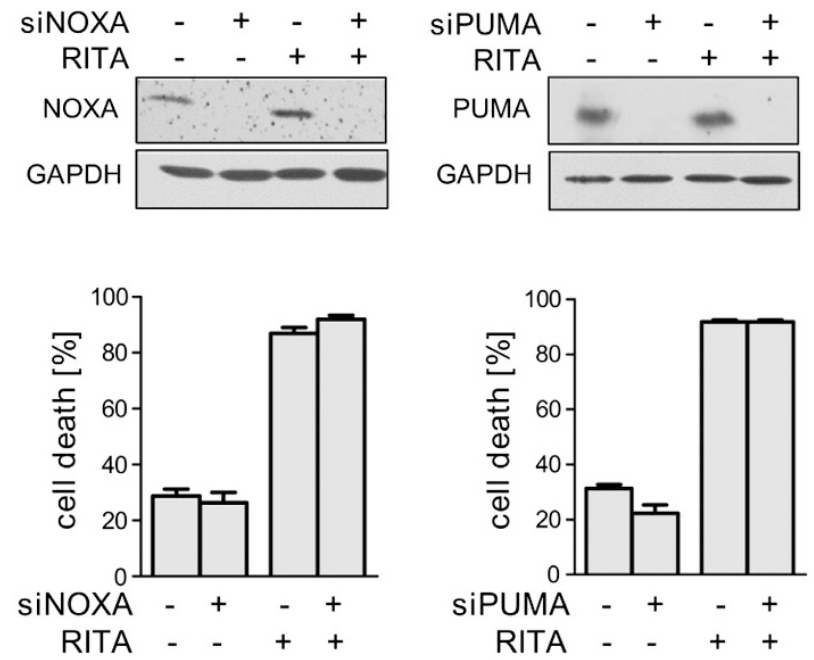

b

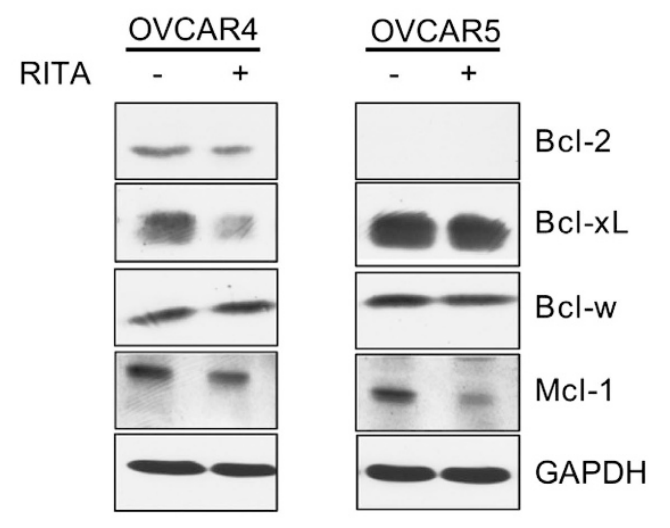

C

OVCAR4

OVCAR5

OVCAR8

SKOV3
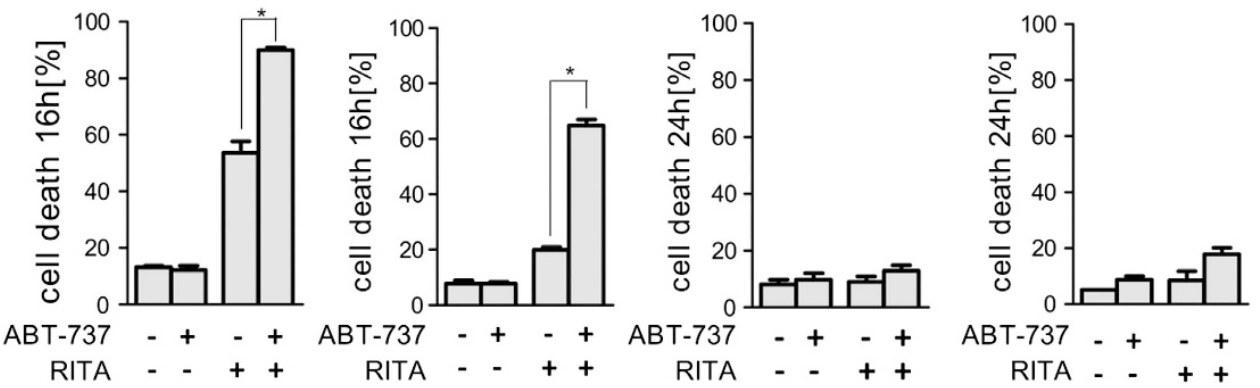

Figure 6 Involvement of BCL-2 family proteins in RITA-mediated apoptosis. (a) Role of the pro-apoptotic NOXA and PUMA for RITA-induced MOMP. The ovarian carcinoma cell line OVCAR4 harboring mtp53 was used for NOXA and PUMA knockdown experiments. Upper panel: representative NOXA (left panel) and PUMA (right panel) western blot analysis demonstrating knockdown efficacy upon siRNA treatment. Lower panel: after pre-incubation with/without indicated siRNAs, cells were incubated with/ without RITA for $48 \mathrm{~h}$ and analyzed for MOMP using TMRM and flow cytometry. Values reflect means \pm S.D. from three independent experiments. (b) Effect of RITA on antiapoptotic BCL-2 family protein levels. Representative western blot experiment showing protein levels of anti-apoptotic BCL-2 family members in cells incubated with/without RITA for $8 \mathrm{~h}$. (c) The BH3 mimetic ABT-737 acts synergistically with RITA in RITA-sensitive ovarian cancer cell lines defective for p53. Cells were incubated with ABT-737 $(1 \mu \mathrm{M})$ and RITA $(1 \mu \mathrm{M})$. After $16 \mathrm{~h}$ (OVCAR4 and OVCAR5) or $24 \mathrm{~h}$ (OVCAR8 and SKOV3), cells were analyzed for MOMP by staining with TMRM and flow cytometry. Values reflect means \pm S.D. from three experiments

lines, all major genotypes of p53 were found. In accordance with the functional outcome, the RITA-mediated regulation of 45 bona fide $\mathrm{p} 53$ target genes also revealed two distinct cell line clusters. Consistent with previous reports, ${ }^{19,25,31}$ RITAinduced apoptosis was dependent on p53 in cells harboring wtp53. In contrast, in the two RITA-sensitive cell lines harboring mtp53, OVCAR3 and OVCAR4, an efficient p53 knockdown did not reduce RITA-mediated apoptosis. Importantly, our results also show that even p53-null cells do have the ability to efficiently induce apoptosis upon RITA. Of note, RITA treatment had significant effects on the expression of 13 of 45 p53 targets investigated in p53-deficient cells. 


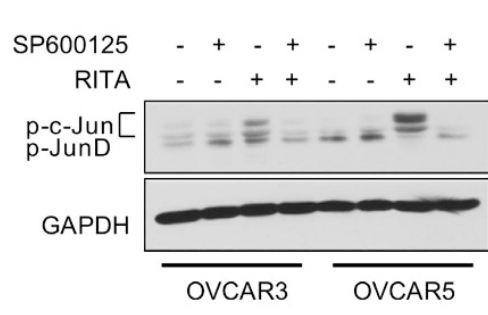

b
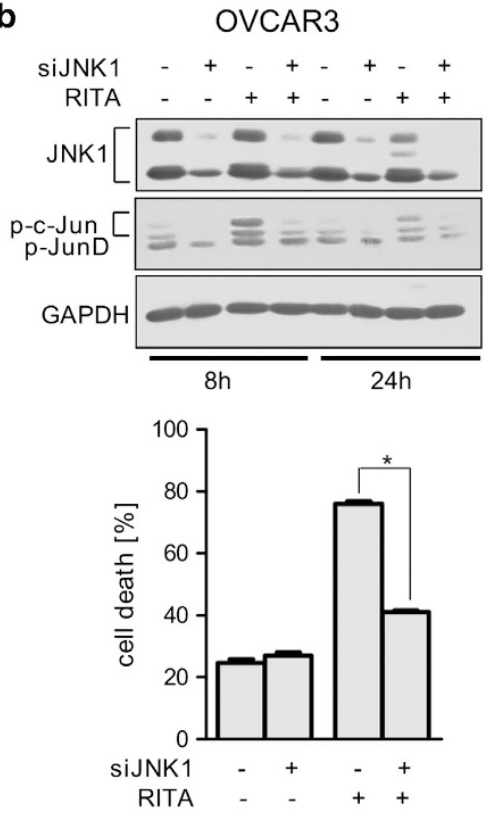

c

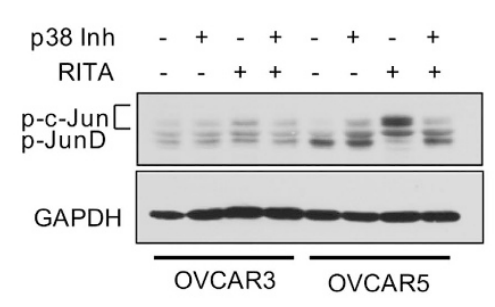

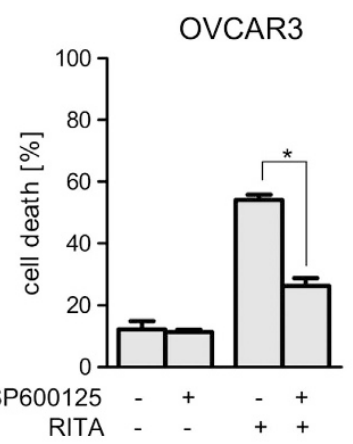

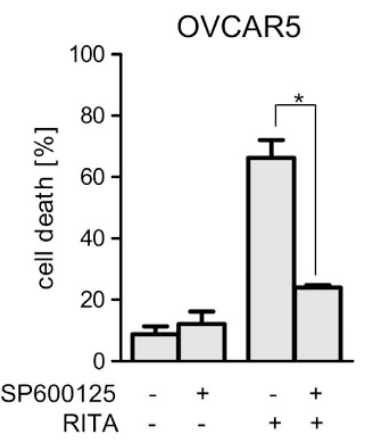

OVCAR5
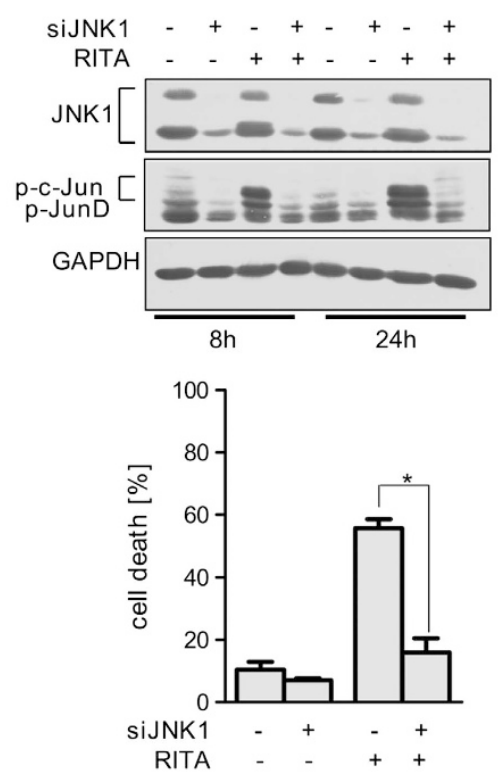

OVCAR3

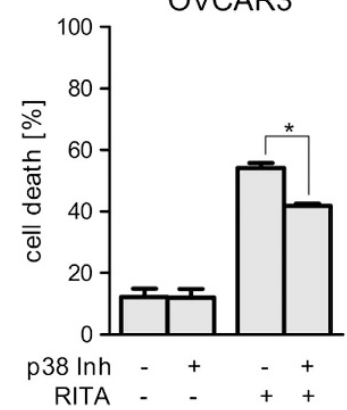

OVCAR5

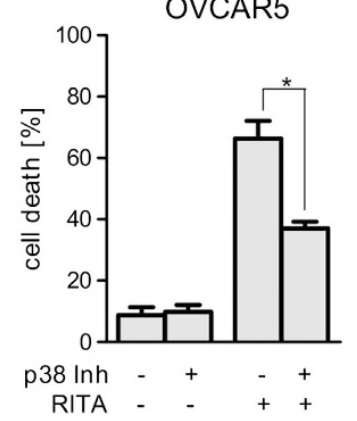

Figure 7 RITA can induce cell death via JNK/SAPK and p38 in mtp53 and p53-null ovarian cancer cells. (a) Role of the JNK/SAPK pathway on RITA-induced cell death in ovarian cancer cell lines harboring mtp53 (OVCAR3) and p53-null cells (OVCAR5). Upper panel: inhibition of JNK was performed by pre-incubation with SP600125 (5 $\mu \mathrm{M}) 2 \mathrm{~h}$ before RITA treatment in OVCAR3 and OVCAR5 cells. Cells were harvested after $3 \mathrm{~h}$ for the detection of c-JUN phosphorylation by western blot analysis (left) and $24 \mathrm{~h}$ for evaluation of cell death by staining with AnnexinV-FITC/PI. Graphs reflect means \pm S.D. from three experiments. (b) Specific silencing of JNK1 is sufficient to significantly reduce RITA-induced cell death in mtp53 OVCAR3 and p53-null OVCAR5 cells. Cells were pre-incubated with specific siRNAs directed against JNK1 $48 \mathrm{~h}$ before RITA treatment for $8 \mathrm{~h}$ and $24 \mathrm{~h}$ for detection of JNK1 protein and c-JUN phosphorylation in OVCAR3 and OVCAR5 cells and $24 \mathrm{~h}$ for evaluation of cell death by staining with AnnexinV-FITC/PI. Graphs reflect means \pm S.D. from three experiments. (c) Role of the p38 pathway for RITA-induced apoptosis. Inhibition of p38 was performed using a specific p38 inhibitor (p38 inhibitor III; $1 \mu \mathrm{M}$ ). After a 2-h pre-incubation, cells were treated with RITA for another $24 \mathrm{~h}$. Efficacy of inhibition was evaluated by western blot analysis of phosphorylated c-JUN (left). OVCAR3 and OVCAR5 cells were stained with AnnexinV-FITC/PI and analyzed by flow cytometry. Values reflect means \pm S.D. from three experiments

This differential regulation was not only independent of p53 but also independent of $\mathrm{p} 63$ and p73. Although the proapoptotic BCL-2 family members NOXA and PUMA were upon the most significantly upregulated genes, we found only minor changes in their protein levels upon RITA treatment. Moreover, silencing of both genes had no effect 
on RITA-induced apoptosis. In addition, knockdown of ATF3 and FOS, the two top-regulated genes upon RITA, also failed to support a significant role of transcriptional regulation of p53 target genes for RITA-induced apoptosis (data not shown). Therefore, neither members of the p53 protein family nor the here investigated differentially regulated pro-apoptotic p53 target genes are involved in RITA-induced apoptosis in p53deficient ovarian cancer cell lines.

These data demonstrate that in addition to the known p53-dependent mechanism of RITA-induced cell death, ${ }^{19-21,25-27,32-34}$ RITA can activate p53-independent pathways leading to apoptosis. In fact, it has been demonstrated that RITA can induce DNA damage ${ }^{35-37}$ and interacts with thioreduxin reductase 1 (TrxR1) leading to ROS induction. ${ }^{33}$ The latter is confirmed by our results showing a significantly higher induction of ROS in RITA-sensitive cell lines from our panel (Supplementary Figure 4). Our results further demonstrated an important role of JNK/SAPK for sensitivity of cells harboring mutant or no p53 protein. JNK/SAPK is activated by a variety of cellular stresses such as ROS, ${ }^{38} \mathrm{UV}$ and ionizing radiation, ${ }^{39,40}$ Cisplatin $^{41}$ and ER stress. ${ }^{42}$ Importantly, the JNK/SAPK pathway has also been shown to be involved in RITA-induced apoptosis in wtp53 cells. ${ }^{31,43}$ These authors hypothesized a positive feedback loop between wtp53 and JNK/SAPK explaining a possible activation of p53 through JNK/SAPK, which then induces a p53-dependent apoptosis. Our data demonstrate that RITA-mediated activation of JNK/SAPK can also lead to significant cell death independently of p53. The mechanism of how these pathways contribute to apoptosis in p53-deficient cells is not entirely clear. Both JNK/SAPK as well as p38 led to a significant induction of c-JUN phosphorylation upon RITA treatment in mtp53 and p53-null cells. Together with FOS, c-JUN is a key component of the AP-1 transcription factor complex and phosphorylation by JNK has been shown to potentiate the transcriptional capacity of this complex. ${ }^{44}$ Importantly, FOS turned out to be one of the most significantly upregulated genes upon RITA. However, neither silencing of $c$-JUN nor FOS had any effect on RITA-induced cell death in p53-deficient ovarian carcinoma cells (data not shown), indicating that JNK-mediated activation of c-JUN and concomitant AP-1-mediated transcriptional regulation of pro-apoptotic genes is not required for RITA-induced cell death in these cells. Besides the regulation of transcription factors, JNKs can also translocate to the mitochondria and directly modulate pro- and anti-apoptotic BCL-2 family proteins. ${ }^{45}$ For example, JNKs specifically phosphorylate BCL-2, BIM and BMF which then activate BAX and BAK to initiate MOMP and apoptosis. ${ }^{46-48}$ Indeed, our data show that RITA-induced cell death in p53-deficient cells strictly depends on MOMP. Consequently, silencing of $B A X$ and $B A K$ also completely abolished apoptosis in RITA-treated OVCAR4 and OVCAR5 cells.

One of the most significantly downregulated gene upon RITA codes for the anti-apoptotic BCL-2 protein, which is in accordance to previous reports performed with cells harboring wtp53. ${ }^{27}$ Of note, this transcriptional downregulation was still seen in OVCAR3 and OVCAR5 cells after silencing of JNK1, which significantly rescued cells from RITA-induced apoptosis indicating that transcriptional regulation of this anti-apoptotic gene is not necessary for RITA-mediated cell death
(Supplementary Figure 5). On protein levels, we also identified other anti-apoptotic BCL-2 proteins to be downregulated after RITA treatment such as MCL-1 (Figure 6b), a finding that was also observed by others in wtp53 cells. ${ }^{21,23,26,27,31}$ Interestingly, again, silencing of JNK1 had no significant effect on RITA-mediated reduction of these antiapoptotic proteins (data not shown). These findings indicate two independent routes by which RITA renders p53-deficient cells prone to apoptosis independently of p53: (i) activation of the JNK/p38 pathway and (ii) downmodulation of antiapoptotic Bcl-2 family members by a yet unknown mechanism. The first might lead to a direct activation of BAX/BAK and concomitant induction of MOMP; the second might facilitate the first but also render these cells hypersensitive toward $\mathrm{BH} 3$ mimetics such as ABT-737 (Figure 6). For MEFs as well as for cancer cell lines, it has been demonstrated that the MCL-1/ NOXA ratio is an important determinant of sensitivity to ABT$737 .{ }^{49}$ Even if NOXA is only slightly upregulated upon RITA on protein level (Figure 6a), there is a shift toward a more proapoptotic MCL-1/NOXA ratio due to RITA-mediated downregulation of $\mathrm{MCL}-1$ in these cells (Figure $6 \mathrm{~b}$ ). This may explain the rapid and very effective induction of cell death observed in RITA-sensitive cells upon combined treatment with RITA and ABT-737 (Figure 6c).

Another interesting finding of the present work is that nonmalignant cells (fibroblasts and PBMNC) were substantially less sensitive to RITA than tumor cells, making RITA an interesting tumor-selective drug. This is in agreement with Saha et al. ${ }^{23}$ showing that despite the expression of wtp53, RITA has only marginal effects on induction of apoptosis in nonmalignant cells. Regardless if RITA may act through induction of ROS and DNA damage and subsequent activation of the p38/SAPK pathways or if other pathways and effects play additional roles, the question still remains open why different cells respond differently. Previous studies indicate that this might be due to a differential uptake and consequently different intracellular RITA concentrations. ${ }^{50}$ The hypothesis that intracellular RITA concentration might be an important determinant of the observed differences in RITA sensitivity is supported by our observation that significantly fewer genes are differentially regulated upon RITA treatment in RITA-resistant cells (Figure 2b). Interestingly, both RITA and Nultin-3 led to a similar accumulation of p53 in wtp53expressing cancer cells characterized by a high sensitivity. ${ }^{32}$ If RITA inhibited p53/MDM2 interaction as efficient as Nutlin-3, then one should indeed expect p53 to accumulate comparably in all wtp53-expressing cells. However, in PBMNC from healthy volunteers characterized by low sensitivity to RITA, we found a marked reduced accumulation of p53 upon RITA compared with that after Nutlin-3 treatment (Supplementary Figure 6), further supporting the hypothesis that RITA concentration in these cells may not be sufficient to effectively accumulate p53 and induce cell death. Nothing is known regarding RITA transport. Differentially regulated transporters important for uptake or efflux of RITA might serve as predictive markers for RITA sensitivity. This is a potential matter of further investigation.

In conclusion, our data demonstrate that RITA is capable to induce cell death not only in cells harboring wtp53 and mtp53 but also in p53-null cells. Importantly, whereas p53 seems to 
have a central role for RITA-mediated effects in wtp53 cells, neither p53 nor p63 and p73 were essential for the RITA response in p53-deficient cells. In these cells, RITA induced a caspase-dependent mitochondrial cell death predominantly mediated by the JNK/SAPK and p38 MAPK pathways.

\section{Materials and Methods}

Cell culture and primary samples. A2780, IGROV1, OVCAR3, OVCAR4, OVCAR5, OVCAR8 and SKOV3 cells were purchased from the NCl60 cell panel. 2102EP cell line was kindly provided by T. Mueller, Department of Oncology, University of Halle, Germany. NTERA-2D1 cell line was obtained from LGS Standards (Wesel, Germany). A549, H460, Calu-1, H23 and TOV-112D cells were procured from the American Type Culture Collection (ATCC, Manassas, VA, USA). All cells were cultivated in RPMI-1640 (Biochrom, Berlin, Germany) supplemented with $10 \%$ FCS and glutamine.

Peripheral blood mononuclear cells (PBMNCs) and fibroblasts were isolated and cultivated as described. ${ }^{51,52}$ The local ethics committee approved the investigation (project number 159/2011BO2) and informed consent was obtained from the patients.

Reagents. RITA (Cayman Chemicals, Ann Arbor, MI, USA) was used in a final concentration of $1 \mu \mathrm{M}$. Nutlin-3 (Sigma-Aldrich, Munich, Germany) was applied at $10 \mu \mathrm{M}$. The pan-caspase inhibitor ZVAD-FMK (Bachem, Bubendorf, Switzerland) was used at $50 \mu \mathrm{M}$. Inhibition of the MAPK pathway was performed using p38 MAP Kinase Inhibitor III (Calbiochem, La Jolla, CA, USA) at $1 \mu \mathrm{M}$ and JNKinhibitor SP600125 (Sigma-Aldrich) at $5 \mu \mathrm{M}$. The BH3 mimetic ABT-737 (ChemieTek, Indianapolis, IN, USA) was used at $1 \mu \mathrm{M}$.

Detection of apoptosis. To examine apoptotic cell death, the cells were stained for flow cytometric analysis using AnnexinV-FITC (BD Pharmingen, Heidelberg, Germany) and propidium iodide (Sigma-Aldrich) as previously described. ${ }^{53}$

Detection of mitochondrial depolarization. Mitochondrial depolarization was measured using TMRM. ${ }^{54}$ After trypsinization, the cells were incubated with $50 \mathrm{nM}$ TMRM for $20 \mathrm{~min}$ at $37^{\circ} \mathrm{C}$ and subsequent analyzed by flow cytometry.

Measurement of cell proliferation. Analysis of cell proliferation was carried out using BrdU as described recently. ${ }^{55}$

Gene expression analysis. Total RNA was extracted by the mirVana miRNA Isolation Kit (Ambion, Austin, TX, USA) according to the manufacturer's protocol. cDNA was generated using the Revert Aid H Minus First strand cDNA synthesis Kit (Fermentas, St Leon-Rot, Germany). Expression analysis was performed on the BioMark HD System (Fluidigm, South San Fransisco, CA, USA) according to the manufacturer's instructions. TaqMan assays were achieved from Applied Biosystems (Norwalk, CT, USA; Supplementary Table 2).

Data analysis was performed using Genespring 12.5-GX-PA (Agilent Technologies, Santa Clara, CS, USA). After calculating $\Delta \Delta$ Ct-values, cluster analysis was accomplished by Euclidean similarity measurements combined with a complete linkage rule.

Protein expression. Total cell lysates were generated using lysis buffer

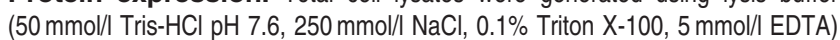
supplemented with complete protease inhibitor cocktail (Roche, Grenzach-Wyhlen, Germany) and PhosSTOP Phosphatase Inhibitor Cocktail (Roche). After sonification and boiling, a specific amount of protein was resuspended in Laemmli and analyzed by western blotting. The primary antibodies used were as follows: anti-p53 (clone: DO-1-\#sc-126, Pab1801-\#sc-98), anti-p63 (clone: 4A4-\#sc-8431) (Santa-Cruz Biotechnology, Heidelberg, Germany); anti-BAX (polyclonal-\#2772), anti-BAK (polyclonal-\#3814), anti-JNK1 (clone: 2C6\#3708) (Cell Signaling, Denvers, MA, USA); anti-p73 (polyclonal-\#ab14430) (Abcam, Cambridge, UK); anti-phospho-c-Jun (Ser73) (polyclonal—\#06-659) (Upstate, Charlottesville, VA, USA).

siRNA Experiments. For silencing of p53, p63, p73, BAX, BAK and JNK1, we used siGenome SMARTpool siRNA (Thermo Fisher Scientific Dharmacon, Lafayette, CO, USA). Target sequences are described in Supplementary Table 4.
As a control, we used siGenome Non-Targeting siRNA \#1 (Thermo Fisher Scientific Dharmacon). In total, $48 \mathrm{~h}$ after transfection using DharmaFECT\#1 (Thermo Fisher Scientific Dharmacon), cells were treated with RITA. The efficacy of silencing was evaluated by western blot or QPCR.

Sequencing. Before sequencing, the synthesized cDNA had first to be amplified by PCR in the p53 gene. The following primers were used:

p53-sense: $5^{\prime}$-GTGACACGCTTCCCTGGAT- $3^{\prime}$

p53-antisense: 5'-CCACAACAAAACACCAGTGC-3'

After electrophoretic separation of the PCR fragment using QIAquick Gel Extraction Kit (Qiagen, Hilden, Germany), the sequencing reaction was performed using the BigDye Terminator v3.1 Cycle Sequencing Kit (Applied Biosystems). Purification of PCR fragments was done through BigDyeXTerminator Purification Kit (Applied Biosystems). Both kits were used according to the manufacturer's instructions.

For Data acquisition, the 3500Dx Genetic Anaylzer (Applied Biosystems) and GeneMapperv4.1 software was used. Sequences were analyzed using Chromas Lite 2.01 (Technelysium, South Brisbane, QLD, Australia).

Statistics. Data were expressed as standard deviation of the means (S.D.). Changes in paired samples were analyzed using two-sided paired $t$-test. $P$-values $<0.05$ were considered significant.

\section{Conflict of Interest}

The authors declare no conflict of interest.

Acknowledgements. We are grateful to Kerstin Willecke for excellent technical assistance. We also thank Thomas Mueller (Department of Oncology, Halle, Germany) for kindly providing the 2102EP cell line. This work was financially supported by the Robert Bosch Foundation (project O2).

\section{Author contributions}

AW designed and performed experiments, analyzed data and wrote the paper; AW and MG performed experiments; MO, WEA, and HvdK designed experiments, analyzed data, wrote the paper and supervised the project. All authors finally approved the paper.

1. Levine AJ, Oren M. The first 30 years of p53: growing ever more complex. Nat Rev Cancer 2009; 9: 749-758

2. Riley $T$, Sontag $E$, Chen $P$, Levine A. Transcriptional control of human p53-regulated genes. Nat Rev Mol Cell Biol 2008; 9: 402-412.

3. Godefroy N, Bouleau S, Gruel G, Renaud F, Rincheval V, Mignotte B et al. Transcriptional repression by $\mathrm{p} 53$ promotes a Bcl-2-insensitive and mitochondria-independent pathway of apoptosis. Nucleic Acids Res 2004; 32: 4480-4490.

4. Mihara M, Erster S, Zaika A, Petrenko O, Chittenden T, Pancoska P et al. p53 has a direct apoptogenic role at the mitochondria. Mol Cell 2003; 11: 577-590.

5. Leu JI-J, Dumont P, Hafey M, Murphy ME, George DL. Mitochondrial p53 activates Bak and causes disruption of a Bak-Mcl1 complex. Nat Cell Biol 2004; 6: 443-450.

6. Chipuk JE, Kuwana T, Bouchier-Hayes L, Droin NM, Newmeyer DD, Schuler M et al. Direct activation of Bax by p53 mediates mitochondrial membrane permeabilization and apoptosis. Science 2004; 303: 1010-1014.

7. Aylon Y, Oren M. p53: guardian of ploidy. Mol Oncol 2011; 5: 315-323.

8. Vousden KH, Prives C. Blinded by the light: the growing complexity of p53. Cell 2009; 137: 413-431.

9. Oren M, Rotter V. Mutant p53 gain-of-function in cancer. Cold Spring Harb Perspect Biol 2010; 2: a001107.

10. Goldstein I, Marcel V, Olivier M, Oren M, Rotter V, Hainaut P et al. Understanding wild-type and mutant p53 activities in human cancer: new landmarks on the way to targeted therapies. Cancer Gene Ther 2011; 18: 2-11.

11. Rivlin N, Brosh R, Oren M, Rotter V. Mutations in the p53 tumor suppressor gene. Genes Cancer 2011; 2: 466-474.

12. Brosh $R$, Rotter $V$. When mutants gain new powers: news from the mutant $p 53$ field. Nat Rev Cancer 2009; 9: 701-713.

13. Wade M, Li Y-C, Wahl GM. MDM2, MDMX and p53 in oncogenesis and cancer therapy. Nat Rev Cancer 2013; 13: 83-96.

14. Stegh AH. Targeting the p53 signaling pathway in cancer therapy-the promises, challenges and perils. Expert Opin Ther Targets 2012; 16: 67-83.

15. Brown CJ, Lain S, Verma CS, Fersht AR, Lane DP. Awakening guardian angels: drugging the p53 pathway. Nat Rev Cancer 2009; 9: 862-873. 
16. Vassilev LT, Vu BT, Graves B, Carvajal D, Podlaski F, Filipovic Z et al. In vivo activation of the p53 pathway by small-molecule antagonists of MDM2. Science 2004; 303: 844-848.

17. Tovar C, Rosinski J, Filipovic Z, Higgins B, Kolinsky K, Hilton H et al. Small-molecule MDM2 antagonists reveal aberrant p53 signaling in cancer: implications for therapy. Proc Natl Acad Sci USA 2006; 103: 1888-1893.

18. Shangary S, Ding K, Qiu S, Nikolovska-Coleska Z, Bauer JA, Liu M et al. Reactivation of p53 by a specific MDM2 antagonist (MI-43) leads to p21-mediated cell cycle arrest and selective cell death in colon cancer. Mol Cancer Ther 2008; 7: 1533-1542.

19. Issaeva N, Bozko P, Enge M, Protopopova M, Verhoef LG, Masucci M et al. Small molecule RITA binds to p53, blocks p53-HDM-2 interaction and activates p53 function in tumors. Nat Med 2004; 10: 1321-1328.

20. Enge M, Bao W, Hedström E, Jackson SP, Moumen A, Selivanova G et al. MDM2-dependent downregulation of p21 and hnRNP $\mathrm{K}$ provides a switch between apoptosis and growth arrest induced by pharmacologically activated p53. Cancer Cell 2009; 15: 171-183.

21. Zhao CY, Grinkevich VV, Nikulenkov F, Bao W, Selivanova G. Rescue of the apoptoticinducing function of mutant p53 by small molecule RITA. Cell Cycle Georget Tex 2010; 9 1847-1855.

22. Gutekunst M, Oren M, Weilbacher A, Dengler MA, Markwardt C, Thomale J et al. p53 hypersensitivity is the predominant mechanism of the unique responsiveness of testicular germ cell tumor (TGCT) cells to cisplatin. PLoS One 2011; 6: e19198.

23. Saha MN, Jiang H, Mukai A, Chang H. RITA inhibits multiple myeloma cell growth through induction of p53-mediated caspase-dependent apoptosis and synergistically enhances nutlin-induced cytotoxic responses. Mol Cancer Ther 2010; 9: 3041-3051.

24. Wei C-L, Wu Q, Vega VB, Chiu KP, Ng P, Zhang T et al. A global map of $p 53$ transcriptionfactor binding sites in the human genome. Cell 2006; 124: 207-219.

25. Zhao CY, Szekely L, Bao W, Selivanova G. Rescue of p53 function by small-molecule RITA in cervical carcinoma by blocking E6-mediated degradation. Cancer Res 2010; 70: 3372-3381.

26. Burmakin M, Shi Y, Hedström E, Kogner P, Selivanova G. Dual targeting of wild type and mutant p53 by small molecule RITA results in the inhibition of N-Myc and key survival oncogenes and kills neuroblastoma cells in vivo and in vitro. Clin Cancer Res 2013; 19 5092-5103.

27. Grinkevich VV, Nikulenkov F, Shi Y, Enge M, Bao W, Maljukova A et al. Ablation of key oncogenic pathways by RITA-reactivated p53 is required for efficient apoptosis. Cancer Cell 2009; 15: 441-453.

28. Dötsch V, Bernassola F, Coutandin D, Candi E, Melino G. p63 and p73, the ancestors of p53. Cold Spring Harb Perspect Biol 2010; 2: a004887.

29. Collavin L, Lunardi A, Del Sal G. p53-family proteins and their regulators: hubs and spokes in tumor suppression. Cell Death Differ 2010; 17: 901-911.

30. Li Y, Prives $\mathrm{C}$. Are interactions with $\mathrm{p} 63$ and $\mathrm{p} 73$ involved in mutant $\mathrm{p} 53$ gain of oncogenic function? Oncogene 2007; 26: 2220-2225

31. Saha MN, Jiang H, Yang Y, Zhu X, Wang X, Schimmer AD et al. Targeting p53 via JNK pathway: a novel role of RITA for apoptotic signaling in multiple myeloma. PLoS One 2012; 7: e30215

32. Rinaldo C, Prodosmo A, Siepi F, Moncada A, Sacchi A, Selivanova G et al. HIPK2 regulation by MDM2 determines tumor cell response to the p53-reactivating drugs nutlin-3 and RITA. Cancer Res 2009; 69: 6241-6248.

33. Hedström E, Eriksson S, Zawacka-Pankau J, Arnér ESJ, Selivanova G. p53-dependent inhibition of TrxR1 contributes to the tumor-specific induction of apoptosis by RITA. Cell Cycle Georget Tex 2009; 8: 3576-3583.

34. Spinnler C, Hedström E, Li H, de Lange J, Nikulenkov F, Teunisse AF et al. Abrogation of Wip1 expression by RITA-activated p53 potentiates apoptosis induction via activation of ATM and inhibition of HdmX. Cell Death Differ 2011; 18: 1736-1745.

35. Yang J, Ahmed A, Poon E, Perusinghe N, de Haven Brandon A, Box G et al Small-Molecule activation of p53 blocks hypoxia-inducible factor $1 \alpha$ and vascular endothelial growth factor expression in vivo and leads to tumor cell apoptosis in normoxia and hypoxia. Mol Cell Biol 2009; 29: 2243-2253.

36. Yang J, Ahmed A, Ashcroft M. Activation of a unique p53-dependent DNA damage response. Cell Cycle Georget Tex 2009; 8: 1630-1632.

37. Ahmed A, Yang J, Maya-Mendoza A, Jackson DA, Ashcroft M. Pharmacological activation of a novel p53-dependent S-phase checkpoint involving CHK-1. Cell Death Dis 2011; 2: e160
38. Shen H-M, Liu Z. JNK signaling pathway is a key modulator in cell death mediated by reactive oxygen and nitrogen species. Free Radic Biol Med 2006; 40: 928-939.

39. Adler V, Schaffer A, Kim J, Dolan L, Ronai Z. UV irradiation and heat shock mediate JNK activation via alternate pathways. J Biol Chem 1995; 270: 26071-26077.

40. Hara T, Namba H, Yang TT, Nagayama Y, Fukata S, Kuma $\mathrm{K}$ et al. lonizing radiation activates c-Jun NH2-terminal kinase (JNK/SAPK) via a PKC-dependent pathway in human thyroid cells. Biochem Biophys Res Commun 1998; 244: 41-44.

41. Sánchez-Perez I, Murguía JR, Perona R. Cisplatin induces a persistent activation of JNK that is related to cell death. Oncogene 1998; 16: 533-540.

42. Lee H, Park MT, Choi BH, Oh ET, Song MJ, Lee $J$ et al. Endoplasmic reticulum stress-induced JNK activation is a critical event leading to mitochondria-mediated cell death caused by $\beta$-lapachone treatment. PLoS One 2011; 6: e21533.

43. Shi Y, Nikulenkov F, Zawacka-Pankau J, Li H, Gabdoulline R, Xu J et al. ROS-dependent activation of JNK converts p53 into an efficient inhibitor of oncogenes leading to robust apoptosis. Cell Death Differ 2014; 21: 612-623.

44. Shaulian E. AP-1-the Jun proteins: oncogenes or tumor suppressors in disguise? Cell Signal 2010; 22: 894-899.

45. Dhanasekaran DN, Reddy EP. JNK signaling in apoptosis. Oncogene 2008; 27 : $6245-6251$

46. Lei K, Davis RJ. JNK phosphorylation of Bim-related members of the Bcl2 family induces Bax-dependent apoptosis. Proc Natl Acad Sci USA 2003; 100: 2432-2437.

47. Srivastava RK, Mi QS, Hardwick JM, Longo DL. Deletion of the loop region of Bcl-2 completely blocks paclitaxel-induced apoptosis. Proc Natl Acad Sci USA 1999; 96: 3775-3780.

48. Yamamoto $\mathrm{K}$, Ichijo $\mathrm{H}$, Korsmeyer SJ. BCL-2 is phosphorylated and inactivated by an ASK1/Jun N-terminal protein kinase pathway normally activated at G(2)/M. Mol Cell Biol 1999; 19: 8469-8478

49. Tromp JM, Geest CR, Breij EC, Elias JA, van Laar J, Luijks DM et al. Tipping the Noxa/Mcl-1 balance overcomes ABT-737 resistance in chronic lymphocytic leukemia. Clin Cancer Res Off J Am Assoc Cancer Res 2012; 18: 487-498.

50. Rivera MI, Stinson SF, Vistica DT, Jorden JL, Kenney S, Sausville EA et al. Selective toxicity of the tricyclic thiophene NSC 652287 in renal carcinoma cell lines: differential accumulation and metabolism. Biochem Pharmacol 1999; 57 : 1283-1295.

51. Fanta S, Sonnenberg M, Skorta I, Duyster J, Miething C, Aulitzky WE et al. Pharmacological inhibition of c-Abl compromises genetic stability and DNA repair in Bcr-Abl-negative cells. Oncogene 2008; 27: 4380-4384.

52. Schmid JO, Dong M, Haubeiss S, Friedel G, Bode S, Grabner A et al. Cancer cells cue the p53 response of cancer-associated fibroblasts to cisplatin. Cancer Res 2012; 72 5824-5832.

53. Skorta I, Oren M, Markwardt C, Gutekunst M, Aulitzky WE, van der Kuip H et al. Imatinib mesylate induces cisplatin hypersensitivity in Bcr-Abl + cells by differential modulation of p53 transcriptional and proapoptotic activity. Cancer Res 2009; 69: 9337-9345.

54. Elmore SP, Nishimura Y, Qian T, Herman B, Lemasters JJ. Discrimination of depolarized from polarized mitochondria by confocal fluorescence resonance energy transfer. Arch Biochem Biophys 2004; 422: 145-152.

55. Haubeiss S, Schmid JO, Mürdter TE, Sonnenberg M, Friedel G, van der Kuip H et al. Dasatinib reverses cancer-associated fibroblasts (CAFs) from primary lung carcinomas to a phenotype comparable to that of normal fibroblasts. Mol Cancer 2010; 9: 168

(2) ()ㅇ Cell Death and Disease is an open-access journal published by Nature Publishing Group. This work is licensed under a Creative Commons Attribution-NonCommercialNoDerivs 3.0 Unported License. The images or other third party material in this article are included in the article's Creative Commons license, unless indicated otherwise in the credit line; if the material is not included under the Creative Commons license, users will need to obtain permission from the license holder to reproduce the material. To view a copy of this license, visit http://creativecommons.org/ licenses/by-nc-nd/3.0/ 